\title{
Modelling and optimizing imperfect maintenance of coatings on steel structures
}

\author{
R. P. Nicolai* \\ Tinbergen \& Econometric Institute \\ Erasmus University Rotterdam \\ J. B. G. Frenk ${ }^{\dagger}$ \\ Econometric Institute \\ Erasmus University Rotterdam \\ R. Dekker $\ddagger$ \\ Econometric Institute \\ Erasmus University Rotterdam
}

Econometric Institute Report 2007-24

\begin{abstract}
Steel structures such as bridges, tanks and pylons are exposed to outdoor weathering conditions. In order to prevent them from corrosion they are protected by an organic coating system. Unfortunately, the coating system itself is also subject to deterioration. Imperfect maintenance actions such as spot repair and repainting can be done to extend the lifetime of the coating. In this paper we consider the problem of finding the set of actions that minimizes the expected maintenance costs over a bounded horizon. To this end we model the size of the area affected by corrosion by a non-stationary gamma process. An imperfect maintenance action is to be done as soon as a fixed threshold is exceeded. The direct effect of such an action on the condition of the coating is assumed to be random. On the other hand, maintenance may also change the parameters of the gamma deterioration process. It is shown that the optimal maintenance decisions related to this problem are a solution of a continuous-time renewal-type dynamic programming equation. To solve this equation time is discretized and it is verified theoretically that this discretization induces only a small error. Finally, the model is illustrated with a numerical example.
\end{abstract}

Keywords: imperfect maintenance, degradation modelling, non-stationary gamma process, renewal-type dynamic programming equation, life-cycle management, condition-based maintenance.

${ }^{*}$ Tinbergen Institute \& Econometric Institute, Erasmus University Rotterdam, P.O. Box 1738, NL-3000 DR Rotterdam, The Netherlands, e-mail: rnicolai@few.eur.nl

${ }^{\dagger}$ Corresponding author. Econometric Institute, Erasmus University Rotterdam, P.O. Box 1738, NL-3000 DR Rotterdam, The Netherlands, e-mail: frenk@ few.eur.nI

${ }^{\ddagger}$ Econometric Institute, Erasmus University Rotterdam, P.O. Box 1738, NL-3000 DR Rotterdam, The Netherlands, e-mail: rdekkerdfew.eur.nl 


\section{Introduction}

Steel structures such as bridges, tanks and pylons are exposed to outdoor weathering conditions. In order to prevent them from corrosion they are protected by organic coating systems. Unfortunately, the coating system itself is also subject to deterioration and after some time the steel loses its coating and starts corroding. Maintenance can be done to improve the condition of the coating system and by doing so the lifetime of the steel structure is also extended. Typical maintenance actions for coating systems are (local) spot repair, repainting and replacement. Spot repair consists of only painting the most visible corroded parts, while repainting means that the entire surface of the structure is repainted without removing the corrosion completely. Finally, in a replacement action the old coating and all corrosion is completely removed and a new coating is applied. Since in spot repair and repainting some corrosion is not removed these actions can be seen as imperfect. Obviously the replacement action restores the condition of the coating to new and is therefore a perfect maintenance action. With respect to costs it is obvious that spot repair is the cheapest action, while replacement is most expensive.

The aim of this study is to find an optimal strategy for imperfect maintenance of engineering structures, in particular steel structures protected by coatings. To this end, we introduce a deterioration model that includes the effect of imperfect maintenance. The actions defined above are then employed to form a maintenance strategy and they are the basis of our optimization model. The objective of this model is to minimize the expected maintenance costs over a bounded horizon. Clearly this bounded horizon is determined by the economic or technical lifetime of the structure. The decision variables are the maintenance actions to be executed.

To model the deterioration process of the coating we use a non-stationary gamma process with state space the size of the coating area affected by corrosion (in e.g. the number of squared meters). As far as the authors know, Abdel-Hameed (1975) was the first to propose the gamma process as a proper model for deterioration in time. The gamma process has increasing sample paths and is therefore a suitable candidate to describe the deterioration of engineering structures, see e.g. Çinlar et al. (1977), van Noortwijk and Klatter (1999), Frangopol et al. (2004) and Newby and Dagg (2004). In particular, in Heutink et al. (2004) and Nicolai et al. (2007) the deterioration of coatings on steel structures is modelled by a non-stationary gamma process. For more examples of the application of gamma processes in maintenance we refer to a recent overview by Van Noortwijk (2007).

Modelling deterioration as a gamma process has also been done in the presence of imperfect maintenance. In Bakker et al. (1999) a lifetime extending maintenance model for engineering structures is introduced. In this model an imperfect maintenance action reduces the amount of deterioration by a fixed amount and after such an action deterioration is again modelled by the same gamma process. However, we shall argue below that the reduction in deterioration may also be random in practice and secondly that the structural parameters of the deterioration process may differ after doing an imperfect maintenance action. Our application thus asks for a more complex model. 
Another stochastic process that is often used to describe deterioration is the Wiener process or Brownian motion (for a definition see Karlin and Taylor, 1975 and for an application in deterioration modelling see Nicolai et al., 2007). In contrast to the gamma process being a jump process with an infinite number of infinitesimal jumps in each finite interval Brownian motion has continuous sample paths. As such, it is the only so-called Lévy process with this property. On the other hand, contrary to the gamma process this process lacks the property of increasing sample paths. Doksum and Hóyland (1992) and Whitmore and Schenkelberg (1997) have proposed the Wiener process as a proper model for deterioration in the context of accelerated degradation due to imperfect maintenance. In both papers the effect of imperfect maintenance is modelled by transforming the time scale of the Wiener process describing deterioration after maintenance.

For steel structures maintenance actions such as spot repair improve the deterioration rate of the coating only locally, whereas other parts of the surface still deteriorate at the same rate. So, as a whole the deterioration process may increase faster after spot repair than after replacement. The same holds for the repainting action. So, we have to extend the model presented in Bakker et al. (1999) and therefore we allow for a structural change in the gamma deterioration process (such as a time transformation) after maintenance is done.

In practice (imperfect) coating maintenance is often done as soon as the area affected by corrosion exceeds a certain intervention level, set by the decision maker. An imperfect maintenance action reduces the size of the affected area by a random amount. This random effect occurs since spot repair and repainting do not cover all corrosion as not all may be visible. Observe all corrosion is removed by a replacement. As the improvement in deterioration is modelled by a nonnegative random variable, the time between two maintenance actions is given by the time the gamma process needs to counterbalance this improvement. This time depends on the parameters of the gamma process and the random improvement. With respect to the latter, we consider generally distributed random improvements in deterioration independent of the gamma process.

Our main interest is in finding the sequence of actions that minimizes the expected maintenance costs over a finite time horizon. This problem can be formulated as a continuous-time renewaltype dynamic programming equation. Time is discretized to solve this equation and it is shown that the solution of the discrete-time problem is close in the supnorm to the solution of the original continuous-time problem. This is supported by numerical evidence.

The outline of this paper is as follows. In Section 2 we introduce a deterioration model for structures subject to imperfect maintenance. The associated continuous-time dynamic programming equation describing the optimal maintenance actions is presented and analyzed in Section 3. Also in this section we give an error analysis due to discretizing this equation. In Section 4 we briefly discuss some techniques presented in Frenk and Nicolai (2007) to compute the cumulative distribution function of the time between two maintenance actions. Next, in Section 5, we employ these techniques to solve the optimization problem formulated in Section 3. In Section 6 we draw conclusions. 


\section{Modelling deterioration and maintenance}

In this section we present a deterioration model for coating systems on corroding structures undergoing imperfect maintenance actions. The deterioration process of the protective coating is given by a (non-stationary) gamma process and maintenance is done as soon as the size of the affected area exceeds a given level $\rho>0$ set by the decision maker. Imperfect maintenance yields a random reduction in this size, bringing it back between 0 and $\rho$. Next, the deterioration process of the coating is again modelled by a, possibly different, non-stationary gamma process. In the remainder of this paper, boldfaced letters are used to denote random variables.

\subsection{Deterioration model}

In this paper the deterioration process of the coating is given by a non-stationary gamma process. To introduce the definition of a gamma process we first observe (Steutel and van Harn, 2004) that the density of a gamma distributed random variable with shape parameter $\beta>0$ and scale parameter $\lambda>0$ is given by

$$
f(x)=\Gamma(\beta)^{-1} \lambda^{\beta} x^{\beta-1} \exp (-\lambda x) 1_{(0, \infty)}(x)
$$

with

$$
\Gamma(\beta):=\int_{0}^{\infty} x^{\beta-1} \exp (-x) \mathrm{d} x
$$

the well-known gamma function. The cumulative distribution function (cdf) of such a random variable is denoted by $\operatorname{gamma}(\beta, \lambda)$. Also we mean by $\mathbf{X} \stackrel{d}{=} \mathbf{Y}$ that the random variables $\mathbf{X}$ and $\mathbf{Y}$ have the same cdf and by $\mathbf{X} \sim F$ that the random variable $\mathbf{X}$ has cdf $F$.

Definition 1 Let $\lambda>0$ and $v:[0, \infty) \rightarrow[0, \infty)$ an increasing, right continuous function satisfying $v(0)=0$. The stochastic process $\mathbf{X}_{v, \lambda}=\left\{\mathbf{X}_{v, \lambda}(t): t \geq 0\right\}$ is called a gamma process with shape function $v$ and scale parameter $\lambda>0$ if

1. $\mathbf{X}_{v, \lambda}(0)=0$ almost surely.

2. The stochastic process $\mathbf{X}_{v, \lambda}$ has independent increments.

3. The random variable $\mathbf{X}_{v, \lambda}(s)-\mathbf{X}_{v, \lambda}(t), s>t$ has a gamma distribution with shape parameter $v(s)-v(t)$ and scale parameter $\lambda>0$.

A gamma process is called stationary if the shape function $v$ is linear. Otherwise it is called non-stationary. A stationary gamma process with shape function $v(t)=t$ and scale parameter 1 will be called standard and for notational convenience such a process is denoted by $\mathbf{X}=\{\mathbf{X}(t)$ : $t \geq 0\}$. In Protter (1992) it is shown that there exists a unique standard gamma process modification with right continuous sample paths having left-hand limits. This is called a càdlàg stochastic 
process and we will always use this modification. Clearly the expectation of the random variable $\mathbf{X}(t)$ is equal to

$$
\mathbb{E}(\mathbf{X}(t))=\lambda^{-1} v(t),
$$

while its variance is given by

$$
\operatorname{Var}(\mathbf{X}(t))=\lambda^{-2} v(t)
$$

To start with our model we assume that for a given strictly increasing continuous shape function $v$ satisfying $v(0)=0$ and $v(\infty)=\infty$ and a given scale parameter $\lambda>0$ the (càdlàg) corrosion process of a new coating is given by a non-stationary gamma process $\mathbf{X}_{v, \lambda}$. After having defined the gamma process we can now formally state for every $t>0$ that

$$
\mathbf{X}_{v, \lambda}(t):=\text { size of the area affected by corrosion at time } t .
$$

\subsection{Effect of maintenance on deterioration}

Having deterioration model (1) at hand, we next introduce a model for the interaction between deterioration and (imperfect) maintenance. A maintenance action $a$ is performed as soon as the size of the affected area exceeds a given level $\rho$. For simplicity it is assumed that any maintenance action takes a negligible amount of time and that such an action is chosen from a finite set $A$ of possible actions. In our specific example we have $A=\{$ spot repair, repainting, replacement $\}$. Introducing for every $r>0$ the hitting time

$$
\mathbf{T}_{v, \lambda}(r):=\inf \left\{t \geq 0: \mathbf{X}_{v, \lambda}(t)>r\right\}
$$

it follows that the random time $\mathbf{L}$ of the first maintenance is given by $\mathbf{T}_{v, \lambda}(\rho)$. Since it can be shown (Frenk and Nicolai, 2007) that

$$
\mathbf{T}_{v, \lambda}(r) \stackrel{d}{=} v^{\leftarrow}(\mathbf{T}(\lambda r))
$$

with $\mathbf{T}(\lambda r)$ denoting the hitting time to level $\lambda r$ of a standard gamma process and $v^{\leftarrow}$ the inverse function of $v$, we obtain that

$$
\mathbf{L} \stackrel{d}{=} v^{\leftarrow}(\mathbf{T}(\lambda \rho))
$$

By relation (2) this yields

$$
\mathbb{P}\{\mathbf{L} \leq t\}=\mathbb{P}\{\mathbf{T}(\lambda \rho) \leq v(t)\}=\mathbb{P}\{\mathbf{X}(v(t))>\lambda \rho\} .
$$

Now consider a maintenance policy $\Pi=\left(a_{i}\right)_{i \in \mathbb{N}}$, where $a_{i} \in A$ denotes the maintenance action chosen after level $\rho$ is exceeded for the $i$ th time. If the selected maintenance action is replacement, then the affected area has size zero again, while for actions belonging to the set $A_{0}=$ \{spot repair, repainting the effect of the maintenance action on the size of the affected area is not known 


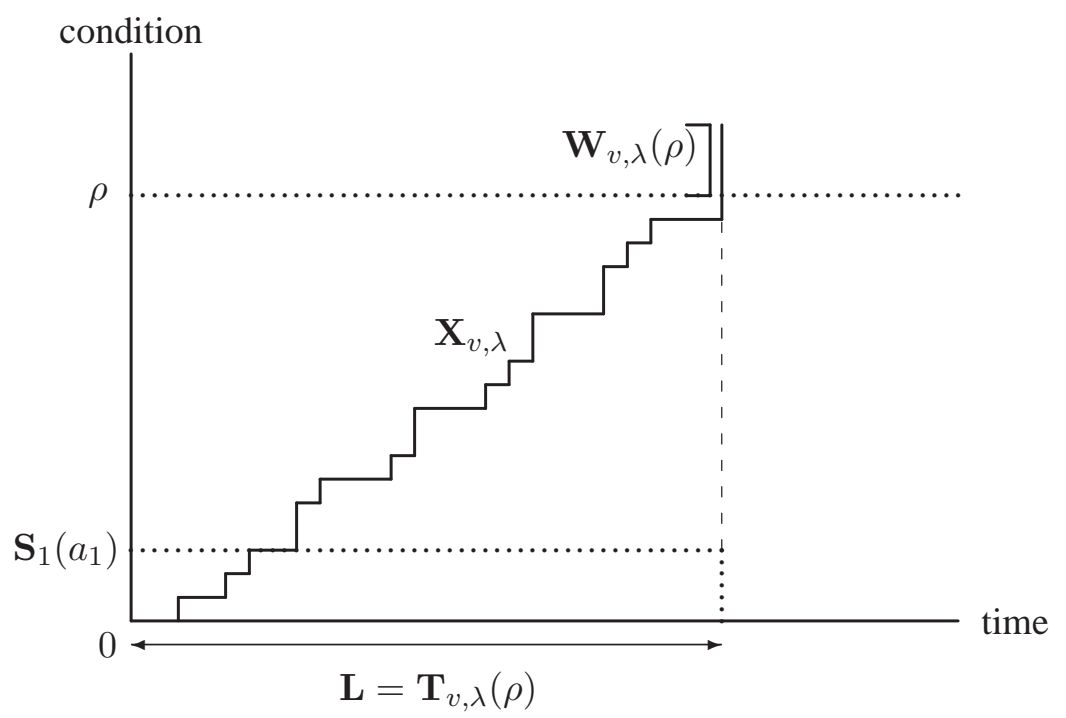

Figure 1: Deterioration path before the first imperfect maintenance action and the immediate effect of this action on deterioration.

beforehand. To model the effect of these imperfect maintenance actions we first observe by the jump discontinuities of the sample paths of a gamma process, that the overshoot

$$
\mathbf{W}_{v, \lambda}(r):=\mathbf{X}_{v, \lambda}\left(\mathbf{T}_{v, \lambda}(r)\right)-r
$$

beyond any level $r>0$ is positive almost surely. Hence the size of the affected area at the first maintenance moment $\mathbf{T}_{v, \lambda}(\rho)$ just before the first maintenance action $a_{1}$ is given by $\rho+\mathbf{W}_{v, \lambda}(\rho)$. This is graphically shown in Figure 1. It is easy to see (Frenk and Nicolai, 2007) that

$$
\mathbf{W}_{v, \lambda}(\rho) \stackrel{d}{=} \lambda^{-1} \mathbf{W}(\lambda \rho)
$$

with $\mathbf{W}(r)$ denoting the overshoot of a standard gamma process at level $r$ and this implies that the random size of the affected area at moment $\mathbf{T}_{v, \rho}(\rho)$ is distributed as $\rho+\lambda^{-1} \mathbf{W}(\lambda \rho)$. In practice $\rho$ is always much larger than the expected overshoot $\lambda^{-1} \mathbb{E}(\mathbf{W}(\lambda \rho))$ (see Appendix A). Hence to avoid complicated mathematical technicalities due to the assumption of a non-stationary gamma deterioration process and its associated discontinuous sample paths it seems realistic from a practical point of view to assume that the effect of any imperfect maintenance action will certainly annihilate the overshoot. Therefore in modelling the effect of an imperfect maintenance action on the size of the area affected by corrosion we will disregard the overshoot. Actually, in other studies the overshoot of the gamma process is often not mentioned at all.

The effect of the first (imperfect) maintenance action $a_{1}$ is now given by a bounded nonnegative random variable $\mathbf{S}_{1}\left(a_{1}\right) \leq \rho$ and this random variable denotes the size of the affected area 
just after performing the first maintenance action $a_{1}$. To model the impact of the first maintenance action we assume that this is represented by the random vector $\mathbf{S}_{1}=\left(\mathbf{S}_{1}(a)\right)_{a \in A}$ consisting of correlated components. Each component $\mathbf{S}_{1}(a)$ within this vector represents the size of the affected area just after performing maintenance action $a$ and this component belongs to $[0, \rho]$. For $a$ representing replacement we obtain $\mathbf{S}_{1}(a)=0$ almost surely, while for $a$ belonging to $A_{0}$ we may also allow the random variable $\mathbf{S}_{1}(a)$ to take the value 0 with a small positive probability. This means that with a (possibly small) probability the effect of the imperfect maintenance actions spot repair or repainting may be the same as the effect of replacement. Since maintenance is always performed at intervention level $\rho$ it follows that the second maintenance action $a_{2}$ in policy $\Pi=\left(a_{i}\right)_{i \in \mathbb{N}}$, is executed at the random time that the deterioration process occurring after the first maintenance action exceeds the (random) level $\rho-\mathbf{S}_{1}\left(a_{1}\right)$. It is assumed that this deterioration process, independent of the previous deterioration gamma process and the (random) improvement of action $a_{1}$, is again a gamma process with continuous strictly increasing shape function $v_{a_{1}}$, satisfying $v_{a_{1}}(0)=0$ and $v_{a_{1}}(\infty)=\infty$, and scale parameter $\lambda_{a_{1}}>0$. Observe the parameters of this process, say $\mathbf{Y}_{1}$, may depend on the first maintenance action $a_{1}$ and on the first maintenance moment. ${ }^{1}$ Hence the second maintenance action occurs at the random time $\mathbf{L}+\mathbf{L}_{1}\left(a_{1}\right)$ with

$$
\mathbf{L}_{1}\left(a_{1}\right)=\mathbf{T}_{v_{a_{1}}, \lambda_{a_{1}}}\left(\rho-\mathbf{S}_{1}\left(a_{1}\right)\right) \stackrel{d}{=} v_{a_{1}}^{\leftarrow}\left(\mathbf{T}^{(1)}\left(\lambda_{a_{1}}\left(\rho-\mathbf{S}_{1}\left(a_{1}\right)\right)\right)\right)
$$

and $\mathbf{T}^{(1)}(r)$ the hitting time to level $r>0$ of an independent copy $\mathbf{X}^{(1)}$ of the standard gamma process $\mathbf{X}$. This shows that the random times $\mathbf{L}$ and $\mathbf{L}_{1}\left(a_{1}\right)$ are independent. Continuing in this way and disregarding the overshoot ${ }^{2}$ of the independent copies $\mathbf{X}^{(i)}, i \in \mathbb{N}$, of a standard gamma process the $n$th maintenance moment associated with policy $\Pi=\left(a_{i}\right)_{i \in \mathbb{N}}$ is distributed as the random variable

$$
\mathbf{L}+\sum_{i=1}^{n-1} \mathbf{L}_{i}
$$

with $\mathbf{L}$ and $\mathbf{L}_{i}, 1 \leq i \leq n-1$, independent nonnegative random variables and

$$
\mathbf{L}_{i}\left(a_{i}\right) \stackrel{d}{=} v_{a_{i}}^{\leftarrow}\left(\mathbf{T}^{(i)}\left(\lambda_{a_{i}}\left(\rho-\mathbf{S}_{i}\left(a_{i}\right)\right)\right)\right)
$$

Clearly in (6) the random variable $\mathbf{T}^{(i)}$ denotes the hitting time of the standard gamma process copy $\mathbf{X}^{(i)}$. Again, it is always assumed (this is necessary for the dynamic programming formulation to be discussed in the next section) that the parameters of the gamma deterioration process occurring between the $i$ th and $(i+1)$ th maintenance action only depend on the $i$ th used maintenance action and the $i$ th maintenance time. In Figure 2 a realization of the second maintenance moment is given. Also it is assumed for every action $a$ and $\mathbf{S}_{i}(a)$ the size of the affected area after performing at the $i$ th maintenance opportunity action $a$ that the random variables $\mathbf{S}_{i}(a), i \in \mathbb{N}$

\footnotetext{
${ }^{1}$ This is suppressed in the notation.

${ }^{2}$ We always assume that in practice the improvement of the condition by even spot repair is sufficiently large. This implies that after each imperfect maintenance action the random amount of corroded area to be counterbalanced is much larger compared to the random overshoot.
} 


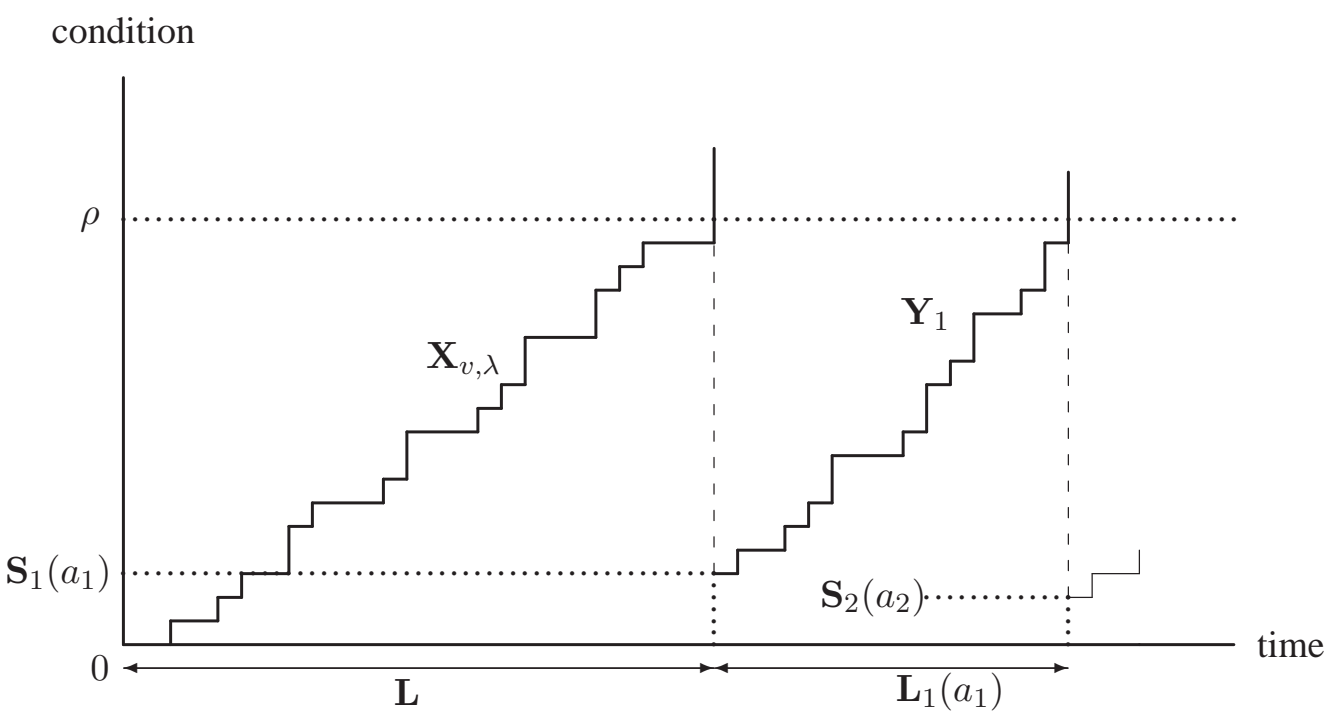

Figure 2: Deterioration paths before and after the first imperfect maintenance action as well as the effects of the first two maintenance actions on deterioration.

are independent and identically distributed. This yields together with the assumption that for each $i \in \mathbb{N}$ the random variable $\mathbf{S}_{i}(a)$ is also independent of $\mathbf{X}^{(i)}$ (and hence $\mathbf{T}^{(i)}$ ), that by relation (6) the random variables $\mathbf{L}_{i}(a), i \in \mathbb{N}$ are independent and identically distributed and

$$
\mathbf{L}_{i}(a) \stackrel{d}{=} v_{a}^{\leftarrow}\left(\mathbf{T}\left(\lambda_{a} \mathbf{R}_{a}\right)\right)
$$

Observe the random variable $\mathbf{R}_{a}:=\rho-\mathbf{S}_{1}(a)$ concentrated on $[0, \rho]$ is independent of the standard gamma process $\mathbf{X}$ and its associated hitting time $\mathbf{T}$. In the remainder of this paper the cdf of the random variables $\mathbf{L}_{i}(a), i \in \mathbb{N}$, is denoted by $F_{a}$ and by relation (7) and $v_{a}$ strictly increasing and continuous satisfying $v_{a}(0)=0$ and $v_{a}(\infty)=\infty$ we obtain

$$
F_{a}(t)=\mathbb{P}\left\{\mathbf{T}\left(\lambda_{a} \mathbf{R}_{a}\right) \leq v_{a}(t)\right\}=\mathbb{P}\left\{\mathbf{X}\left(v_{a}(t)\right)>\lambda_{a} \mathbf{R}_{a}\right\}
$$

Since $v_{a}$ is continuous, $v_{a}(0)=0$ and $\mathbf{R}_{a}$ a positive random variable independent of $\mathbf{X}$ with cdf $G_{\mathbf{R}_{a}}$ satisfying $G_{\mathbf{R}_{a}}(0)=0$ we obtain by the definition of a gamma process that $F_{a}$ is continuous and satisfies $F_{a}(0)=0$. Under some additional condition on the cdf $G_{\mathbf{R}_{a}}$ one can actually show that the cdf $F_{a}$ also satisfies the following inequality.

Definition 2 A function $u:[0, \infty) \rightarrow \mathbb{R}$ is called Lipschitz continuous on the set $B \subseteq[0, \infty)$ with Lipschitz constant $C$ if

$$
|u(t+s)-u(t)| \leq C s
$$

for every $t, t+s \in B$ and $s>0$. 
One can now show the following result.

Lemma 3 If the $c d f G_{\mathbf{R}_{a}}$ for some a $\in A$ is Lipschitz continuous on $[0, \rho]$ with Lipschitz constant $C$, then

$$
0 \leq F_{a}(t+s)-F_{a}(t) \leq C \lambda_{a}^{-1}\left(v_{a}(t+s)-v_{a}(t)\right)
$$

for every $t, s>0$.

Proof. Since the cdf $G_{\mathbf{R}_{a}}$ is Lipschitz continuous on $[0, \rho]$ and $G_{\mathbf{R}_{a}}$ is the cdf of a random variable $\mathbf{R}_{a}$ satisfying $G_{\mathbf{R}_{a}}(\rho)=1$ the cdf $G_{\mathbf{R}_{a}}$ is continuous on $[0, \infty)$. This shows $\mathbb{P}\left\{\mathbf{R}_{a}<x\right\}=$ $\mathbb{P}\left\{\mathbf{R}_{a} \leq x\right\}$ for every $x>0$ and by conditioning on $\mathbf{X}\left(v_{a}(t)\right) \sim F_{v_{a}(t)}$ we obtain from relation (8) that

$$
F_{a}(t)=\int_{0}^{\infty} \mathbb{P}\left\{\mathbf{R}_{a} \leq x \lambda_{a}^{-1}\right\} d F_{v_{a}(t)}(x)=\mathbb{E}\left(G_{\mathbf{R}_{a}}\left(\lambda_{a}^{-1} \mathbf{X}\left(v_{a}(t)\right)\right)\right) .
$$

This implies by the Lipschitz continuity of the $\operatorname{cdf} G_{\mathbf{R}_{a}}$ and $\mathbf{X}$ an increasing process that

$$
\begin{aligned}
0 & \leq F_{a}(t+s)-F_{a}(t) \\
& =\mathbb{E}\left(G_{\mathbf{R}_{a}}\left(\lambda_{a}^{-1} \mathbf{X}\left(v_{a}(t+s)\right)\right)\right)-\mathbb{E}\left(G_{\mathbf{R}_{a}}\left(\lambda_{a}^{-1} \mathbf{X}\left(v_{a}(t)\right)\right)\right) \\
& \leq C \lambda_{a}^{-1} \mathbb{E}\left(\mathbf{X}\left(v_{a}(t+s)\right)-\mathbf{X}\left(v_{a}(t)\right)\right) \\
& =C \lambda_{a}^{-1}\left(v_{a}(t+s)-v_{a}(t)\right)
\end{aligned}
$$

and hence the desired inequality is verified.

For degenerate random variables one can also show the following result.

Lemma 4 It follows for $\mathbf{R}_{a}=\rho$ almost surely that there exists some constant $C>0$ satisfying

$$
F_{a}(t+s)-F_{a}(t) \leq C\left(v_{a}(t+s)-v_{a}(t)\right)
$$

for every $0<t<t+s \leq T$.

Proof. If we consider a gamma process with shape function $v_{a}(t)=t$ for every $t>0$ and scale parameter $\lambda_{a}$ we obtain by relation (8) that

$$
F_{a}(t)=\mathbb{P}\left\{\mathbf{X}(t) \geq \lambda_{a} \rho\right\}=\frac{1}{\Gamma(t)} \int_{\rho}^{\infty} x^{t-1} \exp (-x) d x
$$

It is well known that the function $t \mapsto \Gamma(t)$ is infinitely differentiable on $(0, \infty)$ and $t \Gamma(t)=$ $\Gamma(t+1)$ for every $t>0$ (Rudin, 1976, Whittaker and Watson, 1958). This implies

$$
t \frac{d \Gamma}{d t}(t)+\Gamma(t)=\frac{d \Gamma}{d t}(t+1)
$$


and so

$$
\frac{\frac{d \Gamma}{d t}(t)}{\Gamma(t)^{2}}=\frac{\frac{d \Gamma}{d t}(t+1)}{t \Gamma(t)^{2}}-\frac{1}{t \Gamma(t)}=\frac{\frac{d \Gamma}{d t}(t+1)}{\Gamma(t+1) \Gamma(t)}-\frac{1}{\Gamma(t+1)}
$$

for every $t>0$. Hence we obtain using $\lim _{t \downarrow 0} \Gamma(t)^{-1}=\infty$ that

$$
\lim _{t \downarrow 0} \frac{\frac{d \Gamma}{d t}(t)}{\Gamma(t)^{2}}=-\frac{1}{\Gamma(1)}=-1
$$

Using relation (9) and $t \mapsto \Gamma(t)^{-1}$ is continuously differentiable on $(0, \infty)$ this yields

$$
\sup \left\{\frac{\frac{d \Gamma}{d t}(t)}{\Gamma(t)^{2}}: 0<t \leq T\right\}<\infty
$$

and so the function $t \mapsto \Gamma(t)^{-1}$ has a uniformly bounded derivative on $(0, T]$. A similar observation also applies to the function $t \mapsto \int_{\rho}^{\infty} x^{t-1} \exp (-x) d x$ and both functions $t \mapsto \Gamma(t)^{-1}$ and $t \mapsto \int_{\rho}^{\infty} x^{t-1} \exp (-x) d x$ are therefore Lipschitz continuous on $(0, T]$. Hence the product of these functions is also Lipschitz continuous and we have shown that there exists some $C>0$ satisfying

$$
F_{a}(t+s)-F_{a}(t) \leq C s .
$$

For general shape functions $v_{a}(t)$ it follows again by relation (8) and the result for a standard gamma process given in relation (10) that

$$
F_{a}(t+s)-F_{a}(t) \leq C\left(v_{a}(t+s)-v_{a}(t)\right)
$$

and the result is verified.

Finally we show the following result.

Lemma 5 If the cdf $G_{\mathbf{R}_{a}}$ is Lipschitz continuous on $[0, \rho)$ and the cdf $G_{\mathbf{R}_{a}}$ has a jump discontinuity of size $0<\alpha<1$ at $\rho$, then there exists some $C>0$ satisfying

$$
F_{a}(t+s)-F_{a}(t) \leq C\left(v_{a}(t+s)-v_{a}(t)\right) .
$$

Proof. By relation (8) we obtain

$$
F_{a}(t)=\mathbb{P}\left\{\mathbf{X}\left(v_{a}(t)\right)>\lambda_{a} \mathbf{R}_{a}, \mathbf{R}_{a}<\rho\right\}+\alpha \mathbb{P}\left\{\mathbf{X}\left(v_{a}(t)\right)>\lambda_{a} \rho\right\} .
$$

Observe now that by the continuity of $G_{\mathbf{R}_{a}}$ on $[0, \rho)$ and conditioning on $\mathbf{X}\left(v_{a}(t)\right)$ that

$$
\begin{aligned}
\mathbb{P}\left\{\mathbf{X}\left(v_{a}(t)\right)>\lambda_{a} \mathbf{R}_{a}, \mathbf{R}_{a}<\rho\right\} & =\int_{0}^{\infty} \mathbb{P}\left\{\lambda_{a} \mathbf{R}_{a} \leq \min \left\{x, \lambda_{a} \rho\right\}\right\} d F_{v_{a}}(x) \\
& =\int_{0}^{\infty} G_{\mathbf{R}_{a}}\left(\min \left\{\lambda_{a}^{-1} x, \rho\right\}\right) d F_{v_{a}}(x) \\
& =\mathbb{E}\left(G_{\mathbf{R}_{a}}\left(\min \left\{\lambda_{a}^{-1} \mathbf{X}\left(v_{a}(t)\right), \rho\right\}\right)\right) .
\end{aligned}
$$


Since by our assumption the function $x \mapsto G_{\mathbf{R}_{a}}\left(\min \left\{\lambda_{a}^{-1} x, \rho\right\}\right)$ is Lipschitz continuous on $[0, \infty)$ the desired result follows by applying Lemma 4 to (the second term of) relation (11) and Lemma 3 to relation (12).

In case the function $v_{a}$ is Lipschitz continuous on $[0, T]$ it follows by the above results that the function $F_{a}$ is also Lipschitz continuous on $[0, T]$. Observe Lipschitz continuity turns out to be an important property in showing that the error caused by discretizing the continuous-time renewal-type dynamic programming equation derived in the next section, remains bounded.

\section{Maintenance optimization}

In this section we introduce a finite horizon optimization model for the maintenance of coating systems protecting steel structures. In Section 3.1 the model is formulated as a continuous-time stochastic dynamic programming problem. The corresponding renewal-type optimality equation can only be solved by discretizing time. To this end we propose a simple numerical procedure in Section 3.2 and we investigate in detail the accuracy of this procedure.

\subsection{Continuous-time model}

Let the length of the total planning horizon be denoted by $T$. Observe $T$ represents in practice the finite usage time of the steel structure. The maintenance optimization problem is about which action to select when the deterioration exceeds the fixed intervention level $\rho$. The aim is to minimize the expected maintenance costs over the (finite) planning horizon with respect to the policy $\Pi=\left\{a_{i}\right\}_{i \in \mathbb{N}}$. The cost of a given maintenance action $a$ is denoted by $c(a)$ and it does not depend on (the parameters of) the deterioration process.

To formulate the associated Bellman (optimality) equation of the above dynamic programming problem, let $1_{\mathcal{A}}:[0, T] \rightarrow \mathbb{R}$ be the indicator function of the set $\mathcal{A} \subseteq \mathbb{R}$ given by

$$
1_{\mathcal{A}}(t)= \begin{cases}1 & \text { if } t \in \mathcal{A} \\ 0 & \text { otherwise }\end{cases}
$$

Moreover, denote by $q(t), 0 \leq t \leq T$, the (conditional) minimal expected maintenance cost from time $T-t$ up to time $T$ given that at time $T-t$ a crossing occurs and hence maintenance needs to be done. If this crossing happens for the $i^{*}$ th time and we select at that moment action $a \in A$, then the conditional maintenance cost from time $T-t$ up to time $T$ is given by

$$
c(a) 1_{(0, T]}(t)+q\left(t-\mathbf{L}_{i^{*}}(a)\right) 1_{\left\{\mathbf{L}_{i^{*}}(a) \leq t\right\}} .
$$

To justify relation (13) observe for $\mathbf{L}_{i^{*}}(a)>t$ that the present maintenance action will be the last one and so the total cost from $T-t$ up to $T$ equals $c(a) 1_{(0, T]}(t)$. For $t=0$ we are at the end of the planning horizon and so we do not need to take a maintenance action anymore. This leads 
to zero maintenance cost explaining the indicator function in the above expression. If $\mathbf{L}_{i^{*}}(a) \leq t$ the next maintenance action occurs at time $T-\left(t-\mathbf{L}_{i^{*}}(a)\right)$ and so in this case our total cost from $T-t$ up to $T$ equals $c(a) 1_{(0, T]}(t)+q\left(t-\mathbf{L}_{i^{*}}(a)\right)$. The last term occurs since we need to select from time $T-\left(t-\mathbf{L}_{i^{*}}(a)\right)$ up to $T$ in an optimal way the (possible) remaining actions. By construction the random variables $\mathbf{L}_{i}(a), i \in \mathbb{N}$, are independent and identically distributed and for each $i$ the random variable $\mathbf{L}_{i}(a)$ is also independent of the maintenance costs occurring after the $(i+1)$ th maintenance moment. Hence from relation (13) we obtain that the conditional expected maintenance cost from time $T-t$ up to $T$ given action $a$ is selected equals

$$
c(a) 1_{(0, T]}(t)+\mathbb{E}\left(q\left(t-\mathbf{L}_{i^{*}}(a)\right) \mathbf{1}_{\left\{\mathbf{L}_{i^{*}}(a) \leq t\right\}}\right)=c(a) 1_{(0, T]}(t)+\int_{0}^{t} q(t-y) d F_{a}(y) .
$$

Selecting now at moment $T-t$ the best possible maintenance action we obtain from relation (14) that

$$
q(t)=\min _{a \in A}\left\{c(a) 1_{(0, T]}(t)+\int_{0}^{t} q(t-y) d F_{a}(y)\right\} .
$$

for every $0 \leq t \leq T$. Introducing the convolution operation $*$ given by

$$
\left(q * F_{a}\right)(t):=\int_{0}^{t} q(t-y) d F_{a}(y)
$$

for every $t \geq 0$ relation (15) reduces to

$$
q(t)=\min _{a \in A}\left\{c(a) 1_{(0, T]}(t)+\left(q * F_{a}\right)(t)\right\}
$$

for every $0 \leq t \leq T$. By relation (15) and its definition the optimal value function $q:[0, T] \rightarrow \mathbb{R}$ is increasing, satisfies $q(0)=0$ and has a jump at zero. In the next subsection we will show it is continuous on $(0, T]$. Unfortunately it is difficult to solve the above continuous-time optimality equation and so we need to discretize this renewal-type equation which can be applied to find the optimal maintenance policy.

\subsection{Solving a discrete version of the renewal-type optimality equation}

In this section we solve a discrete version of the continuous-time optimality equation listed in relation (15). In particular, we focus on the (Riemann) lower and upper sums approximation of this renewal-type integral equation. To analyse the error of this approximation let $\mathcal{B}$ denote the set of bounded functions $u:[0, T] \rightarrow \mathbb{R}$ integrable with respect to $F_{a}, a \in A$. If

$$
\|u\|_{T, \infty}:=\sup \{|u(t)|: 0 \leq t \leq T\}
$$

denotes the well-known supnorm on $\mathcal{B}$, the vector space $\left(\mathcal{B},\|\cdot\|_{T, \infty}\right)$ is a Banach space and on this space we introduce the operator $P: \mathcal{B} \rightarrow \mathcal{B}$ given by

$$
P u(t):=\min _{a \in A}\left\{c(a) 1_{(0, T]}(t)+\int_{0}^{t} u(t-y) d F_{a}(y)\right\}
$$


for every $0 \leq t \leq T$. To discretize the above operator let $h>0$ be chosen in such a way that $T=N h$ for some $N \in \mathbb{N}$ and introduce $t_{k}:=k h, k=0, \ldots, N$, and

$$
p_{k}^{(a)}:=F_{a}\left(t_{k+1}\right)-F_{a}\left(t_{k}\right)
$$

for $a \in A$ and $k=0, \ldots, N-1$. If for $i=0,1$ we introduce the set $\mathcal{B}_{i}:=\left\{\left(u\left(t_{i}\right), \ldots, u\left(t_{N}\right)\right)\right.$ : $u \in \mathcal{B}\}$ and this vector space has Chebyshev norm

$$
\|u\|_{i, d}:=\max \left\{\left|u\left(t_{n}\right)\right|: n=i, \ldots, N\right\}
$$

let the operator $U_{h}: \mathcal{B}_{1} \rightarrow \mathcal{B}_{1}$ be given by

$$
U_{h} u\left(t_{n}\right):=\min _{a \in A}\left\{c(a)+\sum_{k=0}^{n-1} u\left(t_{n-k}\right) p_{k}^{(a)}\right\}
$$

for $n=1, \ldots, N$. Also introduce the operator $L_{h}: \mathcal{B}_{0} \rightarrow \mathcal{B}_{0}$ given by

$$
L_{h} u\left(t_{n}\right):=\min _{a \in A}\left\{c(a)+\sum_{k=0}^{n-1} u\left(t_{n-k-1}\right) p_{k}^{(a)}\right\}
$$

for every $n=1, \ldots, N$ and $L_{h} u\left(t_{0}\right)=0$. Before discussing some properties of the above operators we need the following important observation. By relation (8) and $\mathbf{X}$ is a standard gamma process, it follows

$$
F_{a}(T)=\mathbb{P}\left\{\mathbf{X}\left(v_{a}(T)\right)>\lambda_{a} \mathbf{R}_{a}\right\}<1
$$

and since $A$ is a finite action set this implies

$$
\sigma:=\max _{a \in A} F_{a}(T)<1 .
$$

Before mentioning the next result, observe an operator $K$ is called a contraction (with respect to a given norm $\|\|$.$) if there exists some 0<\beta<1$ such that

$$
\|K u-K w\| \leq \beta\|u-w\|
$$

for every $u, v$ belonging to the domain of $K$. The value $0<\beta<1$ is called the contraction number.

Lemma 6 The operator $P: \mathcal{B} \rightarrow \mathcal{B}$ is a contraction (with respect to $\|\cdot\|_{T, \infty}$ ) and its contraction number is given by $0<\sigma<1$. The same holds for the operator $L_{h}: \mathcal{B}_{0} \rightarrow \mathcal{B}_{0}$ with respect to the norm $\|.\|_{0, d}$ and the operator $U_{h}: \mathcal{B}_{1} \rightarrow \mathcal{B}_{1}$ with respect to the norm $\|.\|_{1, d}$ and both contractions have the same contraction number $0<\sigma<1$.

Proof. We only give a proof for the operator $P$, since the proof for the other operators is similar. Let $u, w \in \mathcal{B}$ be given and $0 \leq t \leq T$ fixed. If $a_{w} \in \arg \min _{a \in A}\left\{c(a)+\int_{0}^{t} w(t-y) d F_{a}(y)\right\}$ it follows by the definition of $P$ that

$$
P u(t)-P w(t) \leq \int_{0}^{t}(u-w)(t-y) d F_{a_{w}}(y) .
$$


Also, for $a_{u} \in \arg \min _{a \in A}\left\{c(a)+\int_{0}^{t} u(t-y) d F_{a}(y)\right\}$ we obtain similarly

$$
P u(t)-P w(t) \geq \int_{0}^{t}(u-w)(t-y) d F_{a_{u}}(y) .
$$

By relations (20) and (21) and the definition of $\sigma$ given in relation (19) this implies

$$
|P u(t)-P w(t)| \leq\|u-w\|_{T, \infty} \max \left\{F_{a_{w}}(T), F_{a_{u}}(T)\right\} \leq \sigma\|u-w\|_{T, \infty}
$$

Since the above inequality holds for every $0 \leq t \leq T$ we obtain the desired result.

Since $\left(\mathcal{B},\|\cdot\|_{T, \infty}\right)$ is a Banach space it follows by Lemma 6 and the Banach fixed point theorem (Kreyszig, 1978) that the operator $P$ has a unique fixed point $q$ and for every $u \in \mathcal{B}$ the sequence $P^{m} u$ with $P^{m} u:=P\left(P^{m-1} u\right)$ converges in the supnorm to this fixed point $q$. Hence we obtain for every $u \in \mathcal{B}$ that

$$
\lim _{m \uparrow \infty}\left\|P^{m} u-q\right\|_{T, \infty}=0 \text { and } q=P q .
$$

By relation (15) this fixed point $q$ represents the optimal value function of our dynamic program. For the operator $L_{h}$ and $U_{h}$ we obtain similarly

$$
\lim _{m \uparrow \infty} L_{h}^{m} u=\underline{q} \text { and } \lim _{m \uparrow \infty} U_{h}^{m} u=\bar{q}
$$

with $q$, respectively $\bar{q}$, the unique fixed point of the operator $L_{h}$, respectively $U_{h}$. This means $\underline{q}\left(t_{n}\right)=L_{h} \underline{q}\left(t_{n}\right)$ for every $n=0, \ldots, N$ and $\bar{q}\left(t_{n}\right)=U_{h} \bar{q}\left(t_{n}\right)$ for every $n=1, \ldots, N$. Observe the fixed point $\underline{q}$ of the operator $L_{h}$ is easy to compute by forward substitution. Also at the end of this section we show how to compute $\bar{q}$. Using relations (22) and (23) it is easy to show the following result.

Lemma 7 For every $n=1, \ldots, N$ it follows $q\left(t_{n}\right) \leq q\left(t_{n}\right) \leq \bar{q}\left(t_{n}\right)$.

Proof. Let $u \in \mathcal{B}$ be increasing. Since $u$ is increasing we obtain for every $n=1, \ldots, N$ that

$$
\begin{aligned}
\int_{0}^{t_{n}} u\left(t_{n}-y\right) d F_{a}(y) & =\sum_{k=0}^{n-1} \int_{t_{k}}^{t_{k+1}} u\left(t_{n}-y\right) d F_{a}(y) \\
& \leq \sum_{k=0}^{n-1} u\left(t_{n}-t_{k}\right) p_{k}^{(a)} \\
& =\sum_{k=0}^{n-1} u\left(t_{n-k}\right) p_{k}^{(a)} .
\end{aligned}
$$

This shows by the definition of $P$ and $U_{h}$ that

$$
P u\left(t_{n}\right) \leq U_{h} u\left(t_{n}\right)
$$

for every $n=1, \ldots, N$. Suppose now by induction that $P^{m} u\left(t_{n}\right) \leq U_{h}^{m} u\left(t_{n}\right)$ for some $m \in \mathbb{N}$ and $n=1, \ldots, N$. Since it is easy to verify that $t \rightarrow P^{m} u(t)$ is increasing for every increasing 
$u \in \mathcal{B}$ this implies

$$
\begin{aligned}
P^{m+1} u\left(t_{n}\right) & =\min _{a \in A}\left\{c(a)+\int_{0}^{t_{n}} P^{m} u\left(t_{n}-y\right) d F_{a}(y)\right\} \\
& =\min _{a \in A}\left\{c(a)+\sum_{k=0}^{n-1} \int_{t_{k}}^{t_{k+1}} P^{m} u\left(t_{n}-y\right) d F_{a}(y)\right\} \\
& \leq \min _{a \in A}\left\{c(a)+\sum_{k=0}^{n-1} P^{m} u\left(t_{n-k}\right) p_{k}^{(a)}\right\} \\
& \leq \min _{a \in A}\left\{c(a)+\sum_{k=0}^{n-1} U_{h}^{m} u\left(t_{n-k}\right) p_{k}^{(a)}\right\} \\
& =U_{h}^{m+1} u\left(t_{n}\right) .
\end{aligned}
$$

Hence we have verified that $P^{m} u\left(t_{n}\right) \leq U_{h}^{m} u\left(t_{n}\right)$ for every $m \in \mathbb{N}$ and $n=1, \ldots, N$. This implies by relations (22) and (23) that

$$
q\left(t_{n}\right)=\lim _{m \uparrow \infty} P^{m} u\left(t_{n}\right) \leq \lim _{m \uparrow \infty} U_{h}^{m} u\left(t_{n}\right)=\bar{q}\left(t_{n}\right) .
$$

By a similar proof one can show that $q\left(t_{n}\right) \geq \underline{q}\left(t_{n}\right)$ for $n=1, \ldots, N$ and the result is verified.

In the next result we show that the fixed point $q$ of the operator $P$ is a continuous function on $(0, \mathrm{~T}]$ and under some additional condition even Lipschitz continuous on this set. Observe Lipschitz continuity of the fixed point $q$ on $(0, T]$ is helpful in determining an upper bound on the discretization error.

Lemma 8 The fixed point $q$ of the operator $P$ is continuous on $(0, T]$ and has a jump discontinuity at 0 . Moreover, if $F_{a}$ is Lipschitz continuous on $[0, T]$ for every $a \in A$, then $q$ is also Lipschitz continuous on $(0, T]$.

Proof. Since the function $q$ is increasing it is sufficient to construct an upper bound on $q(t+s)-q(t)$ with $0<t<t+s \leq T$. To start the proof introduce for every increasing and bounded function $u:[0, \infty) \rightarrow[0, \infty)$

$$
d_{s}(u):=\sup _{0<x \leq T-s}\{u(x+s)-u(x)\} .
$$

By the definition of the operator $P, q=P q$ and $q$ increasing we obtain for every $0<t \leq T-s$ fixed that there exists some $a(t) \in A$ (possibly depending on $t$ ) satisfying

$$
\begin{aligned}
q(t+s)-q(t) & \leq \int_{0}^{t}(q(t+s-y)-q(t-y)) d F_{a(t)}(y)+\int_{t}^{t+s} q(t+s-y) d F_{a(t)}(y) \\
& \leq \int_{0}^{t}(q(t+s-y)-q(t-y)) d F_{a(t)}(y)+q(s) \max _{a \in A}\left\{d_{s}\left(F_{a}\right)\right\} .
\end{aligned}
$$

Since by the observation after relation (8) the cdf $F_{a(t)}$ is continuous it follows that

$$
\int_{0}^{t}(q(t+s-y)-q(t-y)) d F_{a(t)}(y)=\lim _{p \uparrow 0} \int_{0}^{t-p}(q(t+s-y)-q(t-y)) d F_{a(t)}(y) .
$$


Using $0<t<T-s$ and relation (24) we obtain for every $p>0$ that

$$
\int_{0}^{t-p}(q(t+s-y)-q(t-y)) d F_{a(t)}(y) \leq d_{s}(q) F_{a(t)}(T) .
$$

Hence by relations (26) and (19)

$$
\int_{0}^{t}(q(t+s-y)-q(t-y)) d F_{a(t)}(y) \leq \sigma d_{s}(q) .
$$

Using the above inequality and relation (25) yields

$$
q(t+s)-q(t) \leq \sigma d_{s}(q)+q(s) \max _{a \in A}\left\{d_{s}\left(F_{a}\right)\right\}
$$

Since relation (27) holds for every $0<t<t+s \leq T$, we finally obtain that

$$
d_{s}(q) \leq \sigma d_{s}(q)+q(s) \max _{a \in A}\left\{d_{s}\left(F_{a}\right)\right\} .
$$

By relation 19)) we know that $\sigma<1$ and so

$$
d_{s}(q) \leq \frac{q(s) \max _{a \in A}\left\{d_{s}\left(F_{a}\right)\right\}}{1-\sigma} .
$$

Since the cdf $F_{a}$ is continuous on $[0, \infty)$ and hence uniformly continuous on $[0, T]$ (Rudin, 1976), implying

$$
\lim _{s \downarrow 0} d_{s}\left(F_{a}\right)=0
$$

for every $a \in A$, the continuity of $q$ on $(0, T)$ follows by relation (28) and $A$ finite. For $F_{a}, a \in A$, Lipschitz continuous we obtain for every $a \in A$ that there exists some finite $C_{a}>0$ satisfying

$$
d_{s}\left(F_{a}\right) \leq C_{a} s
$$

Again by relation (28) and $A$ finite the Lipschitz continuity of $q$ on $(0, T]$ follows.

In the next lemma we give a conservative bound on the error $\|q-\underline{q}\|_{1, d}$ if the cdfs $F_{a}, a \in A$, are Lipschitz continuous.

Lemma 9 If the cdf $F_{a}$ is Lipschitz continuous for every $a \in A$, then then there exists some constant $C>0$ independent of $h$ such that

$$
\|q-\underline{q}\|_{1, d} \leq C h .
$$


Proof. By the triangle inequality and $q$, respectively $\underline{q}$, is a fixed point of the operator $P$, respectively $L_{h}$, we obtain

$$
\begin{aligned}
\|q-\underline{q}\|_{1, d} & =\left\|P q-L_{h} \underline{q}\right\|_{1, d} \\
& \leq\left\|P q-L_{h} q\right\|_{1, d}+\left\|L_{h} q-L_{h} \underline{q}\right\|_{1, d} .
\end{aligned}
$$

Since $F_{a}$ is Lipschitz continuous for every $a \in A$ we obtain by Lemma 8 that the fixed point $q$ is Lipschitz continuous and increasing on $(0, T]$ (remember $q(0)=0$ ) and so there exists some finite positive constant $C_{q}$ satisfying

$$
\left|P q\left(t_{n}\right)-L_{h} q\left(t_{n}\right)\right| \leq C_{q} h+q(h) \max _{a \in A}\left\{p_{n-1}^{(a)}\right\}
$$

for every $n=1, \ldots, N$. Introducing for a cdf $F$ the value

$$
d_{h}(F):=\sup _{k=0, \ldots, N-1}\left\{F\left(t_{k+1}\right)-F\left(t_{k}\right)\right\}
$$

it follows by relation (30) that

$$
\left\|P q-L_{h} q\right\|_{1, d} \leq C_{q} h+q(h) \max _{a \in A}\left\{d_{h}\left(F_{a}\right)\right\} .
$$

Since $L_{h}$ is a contraction with contraction number $0<\sigma<1$ and $q(0)=\underline{q}(0)=0$ this implies by relations (29) and (32) that

$$
\|q-\underline{q}\|_{1, d} \leq C_{q} h+q(h) \max _{a \in A}\left\{d_{h}\left(F_{a}\right)\right\}+\sigma\|q-\underline{q}\|_{1, d}
$$

and so

$$
\|q-\underline{q}\|_{1, d} \leq \frac{C_{q} h+q(h) \max _{a \in A}\left\{d_{h}\left(F_{a}\right)\right\}}{1-\sigma} .
$$

By the Lipschitz continuity of $F_{a}$ with Lipschitz constant $C_{a}$ we obtain $d_{h}\left(F_{a}\right) \leq C_{a} h$. Also by the Lipschitz continuity of $q$ on $(0, T]$ it follows that

$$
q(h)=q(h)-\lim _{t \downarrow 0} q(t)+\min _{a \in A}\{c(a)\} \leq C_{q} h+\min _{a \in A}\{c(a)\}
$$

and this shows in combination with relation (33) and $A$ a finite set the desired result.

It is also possible without any conditions on the cdf $F_{a}$ to obtain an estimate of the supnorm error. Introducing

$$
\nu_{h}:=\max _{1 \leq k \leq N-1}\left\{\underline{q}\left(t_{k+1}\right)-\underline{q}\left(t_{k}\right)\right\}
$$

one can show the following result. 


\section{Lemma 10 It follows that}

$$
\|q-\underline{q}\|_{1, d} \leq\|\bar{q}-\underline{q}\|_{1, d} \leq \frac{\nu_{h}+\underline{q}\left(t_{1}\right) \max _{a \in A}\left\{d_{h}\left(F_{a}\right)\right\}}{1-\sigma}
$$

with $d_{h}\left(F_{a}\right)$ defined in relation (31).

Proof. For every $n=1, . ., N$ that there exists some $a_{n} \in A$ such that

$$
U_{h} \underline{q}\left(t_{n}\right)-L_{h} \underline{q}\left(t_{n}\right) \leq \sum_{k=0}^{n-1} \underline{q}\left(t_{n-k}\right) p_{k}^{\left(a_{n}\right)}-\sum_{k=0}^{n-1} \underline{q}\left(t_{n-k-1}\right) p_{k}^{\left(a_{n}\right)}
$$

Using $\underline{q}(0)=0$ the last term can be rewritten as

$\sum_{k=0}^{n-1} \underline{q}\left(t_{n-k}\right) p_{k}^{\left(a_{n}\right)}-\sum_{k=0}^{n-1} \underline{q}\left(t_{n-k-1}\right) p_{k}^{\left(a_{n}\right)}=\sum_{k=0}^{n-2}\left(\underline{q}\left(t_{n-k}\right)-\underline{q}\left(t_{n-k-1}\right)\right) p_{k}^{\left(a_{n}\right)}+\underline{q}\left(t_{1}\right) p_{n-1}^{\left(a_{n}\right)}$.

Since $\underline{v}$ is increasing (check this by using $\lim _{m \uparrow \infty} L_{h}^{m} u\left(t_{n}\right)=\underline{v}\left(t_{n}\right)$ and $u$ increasing implies $L_{h} u$ increasing) we therefore obtain

$$
U_{h} \underline{q}\left(t_{n}\right)-L_{h} \underline{q}\left(t_{n}\right) \leq \nu_{h}+\underline{q}\left(t_{1}\right) \max _{a \in A}\left\{p_{n-1}^{(a)}\right\} .
$$

This implies

$$
\left\|U_{h} \underline{q}-L_{h} \underline{q}\right\|_{1, d} \leq \nu_{h}+\underline{q}\left(t_{1}\right) \max _{a \in A}\left\{d_{h}\left(F_{a}\right)\right\} .
$$

Also, using $U_{h}$ is a contraction with contraction number $\sigma$, it follows

$$
\begin{aligned}
\left\|U_{h} \bar{q}-L_{h} \underline{q}\right\|_{1, d} & =\left\|U_{h} \bar{q}-U_{h} \underline{q}+U_{h} \underline{q}-L_{h} \underline{q}\right\|_{1, d} \\
& \leq\left\|U_{h} \bar{q}-U_{h} \underline{q}\right\|_{1, d}+\left\|U_{h} \underline{q}-L_{h} \underline{q}\right\|_{1, d} \\
& \leq \sigma\|\bar{q}-\underline{q}\|_{1, d}+\left\|U_{h} \underline{q}-L_{h} \underline{q}\right\|_{1, d} .
\end{aligned}
$$

Combining relations (34) and (35) finally yields

$$
\begin{aligned}
\|\bar{q}-\underline{q}\|_{1, d} & =\left\|U_{h} \bar{q}-L_{h} \underline{q}\right\|_{1, d} \\
& \leq \sigma\|\bar{q}-\underline{q}\|_{1, d}+\nu_{h}+\underline{q}\left(t_{1}\right) \max _{a \in A}\left\{d_{h}\left(F_{a}\right)\right\}
\end{aligned}
$$

and hence

$$
\|\bar{q}-\underline{q}\|_{1, d} \leq \frac{\nu_{h}+\underline{q}\left(t_{1}\right) \max _{a \in A}\left\{d_{h}\left(F_{a}\right)\right\}}{1-\sigma} .
$$

By Lemma 7 it also follows that

$$
\|q-\underline{q}\|_{1, d} \leq\|\bar{q}-\underline{q}\|_{1, d}
$$


and hence by relation (36) we have shown the result.

As mentioned earlier, it is easy to compute the fixed point $\underline{q}$ of the operator $L_{h}$ by forward substitution. Since the right-hand side of equation (17) involves a function evaluation in $t_{n}$, it seems to be more complicated to compute the fixed point $\bar{q}$ of $U_{h}$. However, it turns out that there exists an easy analytic expression for $\bar{q}\left(t_{n}\right), n=1,2, \ldots, N$. To this end we require the following lemma.

Lemma 11 For $A$ a finite set, $f: A \rightarrow[0, \infty)$ and $g: A \rightarrow[0,1)$ the optimality equation

$$
w=\min _{a \in A}\{f(a)+g(a) w\} .
$$

has a unique solution $w \geq 0$ given by

$$
w=\min _{a \in A}\left\{\frac{f(a)}{1-g(a)}\right\} .
$$

Proof. Since $w \mapsto \min _{a \in A}\{f(a)+g(a) w\}$ is a contraction, the above optimality equation has a unique solution. Moreover, by the definition of $w$ we obtain $w \leq f(a)+g(a) w$ for every $a \in A$ and so

$$
w \leq \min _{a \in A}\left\{\frac{f(a)}{1-g(a)}\right\} .
$$

Again by its definition there exists some $a \in A$ such that $w=f(a)+g(a) w$ and the result is proved.

Lemma 12 The fixed point $\bar{q}$ of the operator $U_{h}$ listed in relation (17) is given by

$$
\bar{q}\left(t_{1}\right)=\min _{a \in A}\left\{\frac{c(a)}{1-p_{0}^{(a)}}\right\}
$$

and for $n=2, \ldots, N$

$$
\bar{q}\left(t_{n}\right)=\min _{a \in A}\left\{\frac{c(a)+\sum_{k=1}^{n-1} \bar{q}\left(t_{n-k}\right) p_{k}^{(a)}}{1-p_{0}^{(a)}}\right\} .
$$

Proof. Since $0 \leq p_{0}^{(a)}<1$ for every $a$ and $A$ is a finite set the expression for $\bar{q}\left(t_{1}\right)$ is a direct consequence of Lemma 11. Also by the definition $\bar{q}\left(t_{n}\right)$ we obtain

$$
\bar{q}\left(t_{n}\right)=\min _{a \in A}\left\{c(a)+\sum_{k=1}^{n-1}\left(\bar{q}\left(t_{n-k}\right) p_{k}^{(a)}\right)+p_{0}^{(a)} \bar{q}\left(t_{n}\right)\right\}
$$

for every $n=2, \ldots, N$. Taking $f(a)=c(a)+\sum_{k=1}^{n-1} \bar{q}\left(t_{n-k}\right) p_{k}^{(a)}$ and $g(a)=p_{0}^{(a)}$, the second formula follows again from Lemma 11.

In Section 5 we will compute both $q$ and $\bar{q}$ and this yields by Lemma 7 an upper bound on the 'empirical' accuracy of the discretization procedure(s). Also in Appendix B we list a simple algorithm for computing the fixed points $\underline{q}$ and $\bar{q}$. 


\section{Computing the cdf of the time between two maintenance actions}

To solve the optimization problems introduced in Section 3 we need a fast method to compute the cdf $F_{a}$ listed in relation (8) of the time between two maintenance actions. Unfortunately, this cdf only has a nice analytical expression in some special cases (Frenk and Nicolai (2007)). In general, evaluating this cdf numerically e.g. via its two-dimensional integral representation is time-consuming. However, as we will see it is easy to approximate this cdf.

Note that $F_{a}$ relates to the cdf of the first time a standard gamma process exceeds a random threshold. For notational convenience we suppress the subscript $a$ in this section and consider the cdf $H_{\mathbf{R}}$ given by $H_{\mathbf{R}}(t):=\mathbb{P}\{\mathbf{T}(\mathbf{R}) \leq t\}$ for $t \geq 0$, where $\mathbf{T}(\mathbf{R})$ is the first time a standard gamma process exceeds some nonnegative random variable $\mathbf{R}$. In particular, taking $\mathbf{R}=\lambda_{a} \mathbf{R}_{a}$ and replacing $t$ by $v_{a}(t)$ yields relation (8). In the remainder of this section we focus on the computation of the cdf $H_{\mathbf{R}}$ for nonnegative random variables $\mathbf{R}$ having a general distribution. In Section 4.1 we give expressions for this cdf and in Section 4.2 we demonstrate how the desired hitting time distribution can be approximated.

\subsection{General expressions}

Since the nonnegative, non-defective, random variable $\mathbf{R}$ is by definition independent of the gamma process and its cdf $G_{\mathbf{R}}$ satisfies $G_{\mathbf{R}}(0)=0$ it follows by conditioning on the random variable $\mathbf{R}$ that

$$
H_{\mathbf{R}}(t)=\mathbb{P}\{\mathbf{T}(\mathbf{R}) \leq t\}=\mathbb{P}\{\mathbf{X}(t)>\mathbf{R}\}=\int_{0}^{\infty} \mathbb{P}\{\mathbf{X}(t)>r\} d G_{\mathbf{R}}(r)
$$

for every $t \geq 0$. Moreover, by conditioning on the random variable $\mathbf{X}(t)$ we obtain for $G_{\mathbf{R}}$ a continuous cdf the equivalent representation

$$
H_{\mathbf{R}}(t)=\mathbb{E}\left(G_{\mathbf{R}}(\mathbf{X}(t))\right) .
$$

In general, the above expressions have to be computed via numerical integration. On the other hand, if the random variable $\mathbf{R}$ has a degenerate, uniform or gamma-type distribution, then $H_{\mathbf{R}}$ has a 'nice' analytical expression (Frenk and Nicolai, 2007).

\subsection{A simple approximation}

In Frenk and Nicolai (2007) it is shown empirically and theoretically that a linear interpolation of $H_{\mathbf{R}}$ at the integer points approximates the true cdf quite well in the supnorm. This approximation is given by

$$
H_{\mathbf{R}}(t) \approx(t-\lfloor t\rfloor) H_{\mathbf{R}}(\lfloor t\rfloor+1)+(1-(t-\lfloor t\rfloor)) H_{\mathbf{R}}(\lfloor t\rfloor), t \geq 0 .
$$


To evaluate the continuous cdf $F$ on its integer points one uses that

$$
\begin{aligned}
H_{\mathbf{R}}(n+1)-H_{\mathbf{R}}(n) & =\mathbb{P}\{n<\mathbf{T}(\mathbf{R}) \leq n+1\} \\
& =\mathbb{P}\{\lfloor\mathbf{T}(\mathbf{R})\rfloor=n\} \\
& =\frac{1}{n !} \mathbb{E}\left(\mathbf{R}^{n} \exp (-\mathbf{R})\right)
\end{aligned}
$$

for every $n \in \mathbb{N} \cup\{0\}$. Alternatively, if

$$
\widehat{G}_{\mathbf{R}}(\tau):=\mathbb{E}(\exp (-\tau \mathbf{R}))
$$

is the probability Laplace Stieltjes transform (pLSt) of the cdf $G_{\mathbf{R}}$, then relation (42) is the same as

$$
H_{\mathbf{R}}(n+1)-H_{\mathbf{R}}(n)=\frac{(-1)^{n}}{n !} \widehat{G}_{\mathbf{R}}^{(n)}(1)
$$

for every $n \in \mathbb{N} \cup\{0\}$ with $\widehat{G}_{\mathbf{R}}^{(n)}, n \in \mathbb{N}$, denoting the $n$th derivative of $\widehat{G}_{\mathbf{R}}$ and $\widehat{G}_{\mathbf{R}}^{(0)}:=\widehat{G}_{\mathbf{R}}$. If the derivatives of $\widehat{G}_{\mathbf{R}}$ are elementary functions we can directly apply relation (43). Examples are given by the class of infinitely divisible distributions, including the gamma distribution, (power transformations of) the uniform distribution and the class of concave distributions (for more details, see Frenk and Nicolai, 2007).

Remark 13 Observe the above piecewise linear approximation is derived for a standard gamma process and by construction it is Lipschitz continuous. By relation (3) it can also be used for a non-stationary gamma process. Approximating now the cdf of the time between two maintenance actions $F_{a}$ by $F_{a}^{\text {prox }}$ yields a different continuous-time dynamic programming equation, given by

$$
q_{\text {prox }}(t)=\min _{a \in A}\left\{c(a) 1_{(0, T]}(t)+\int_{0}^{T} q_{\text {prox }}(t-y) d F_{a}^{\text {prox }}(y)\right\} .
$$

If the shape functions $v_{a}$ are Lipschitz continuous on [0,T], it follows by the remark after Lemma 3 that the approximation $F_{a}^{p r o x}$ is clearly Lipschitz continuous and so we may conclude from Lemma 8 that $q_{\text {prox }}$ being the solution of the above approximate Bellman equation is Lipschitz continuous on $(0, T]$ and satisfies $q_{\text {prox }}(0)=0$. Also, since $F_{a}^{\text {prox }}$ is close in the supnorm to $F_{a}$, the same holds for the fixed point $q_{\text {prox }}$ of relation (44) and the fixed point $q$ of relation (15). By the previous results one may therefore conclude that the discretization of the above approximative Bellman equation yields accurate results.

\section{Numerical example}

Let us illustrate the model and the methods discussed in the previous sections with the following numerical example. Consider a planning horizon of at most 50 time units, i.e. $T \in[0,50]$. The 

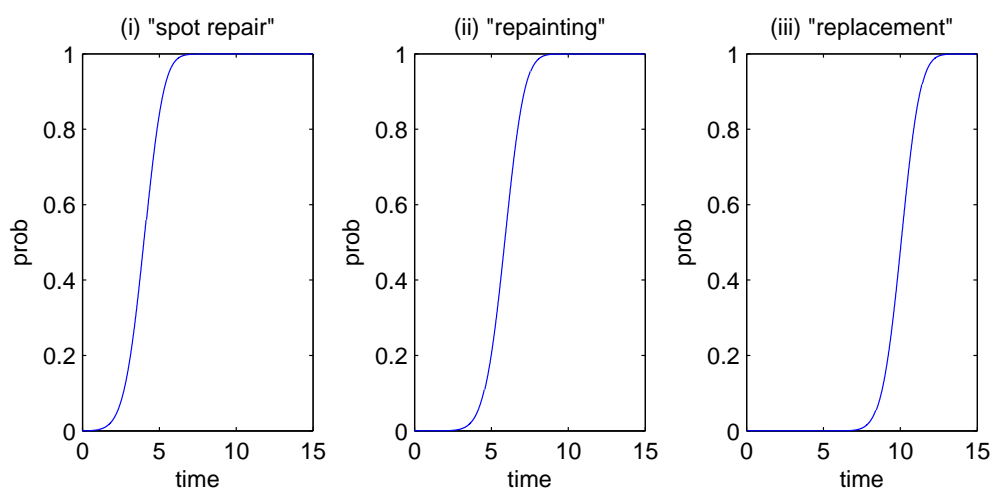

Figure 3: Probability distributions of the time between two maintenance actions: (i) $F_{a_{s}}$, (ii) $F_{a_{r}}$, and (iii) $F_{a_{f}}$.

initial gamma deterioration process is given by $\mathbf{X}_{v, \lambda}$ with $v(t)=0.25 t^{2}$ and $\lambda=1$. The intervention level is given by $\rho=25$. Let $A=\left\{a_{s}, a_{r}, a_{f}\right\}$ be the set of maintenance actions representing spot repair, repainting and full replacement. Suppose the size of the affected area just after each of these three maintenance actions is given by $\mathbf{S}\left(a_{s}\right) \sim \operatorname{unif}(15,20), \mathbf{S}\left(a_{r}\right) \sim \operatorname{unif}(10,15)$ and $\mathbf{S}\left(a_{f}\right)=0$ almost surely, respectively. It follows that the reduction $\mathbf{R}_{a}$ is uniformly distributed for $a \in\left\{a_{s}, a_{r}\right\}$ and $\mathbf{R}_{a_{f}}$ has a degenerate distribution. The cdf $F_{a}$ is computed numerically for all actions $a \in A$ and the corresponding graphs are shown in Figure 3.

The costs associated with the maintenance actions are $c\left(a_{s}\right)=2, c\left(a_{r}\right)=3$ and $c\left(a_{f}\right)=$ 5 , respectively. Finally, let the gamma processes describing the size of the affected area after maintenance action $a$ have shape function $v_{a}=v, \forall a \in A$, and scale parameter $\lambda_{a_{s}}=\lambda / 2$, $\lambda_{a_{r}}=2 \lambda / 3$ and $\lambda_{a_{f}}=\lambda$, respectively. By doing so deterioration is accelerated by a factor $2(3 / 2)$ after spot repair (repainting), while keeping the variability of the deterioration process the same. Moreover, the ratio of the expected time until the next crossing of level $\rho$ due to a maintenance action and the unit cost is approximately 2 for all maintenance actions.

Algorithm 14 in Appendix B has been utilized to compute $q$ and $\bar{q}$ with discretization step $h=0.01$. It appears from plots (a) and (b) in Figure 4 that these bounds on $q$ are approximately equal. That is, in this example the discretization of the dynamic programming equation yields accurate results. In plot (a) we also see that the minimal expected cost (just before a maintenance action has to be selected) as a function of the planning horizon $T$ is almost linear. Plots (c) and (d) in Figure 4 show for both bounds the optimal maintenance action just before the intervention level is exceeded $T$ time units before the end of the horizon. It can be seen that on the short term 'spot repair' is the best action, whereas on the long term 'replacement' is most beneficial. It is 

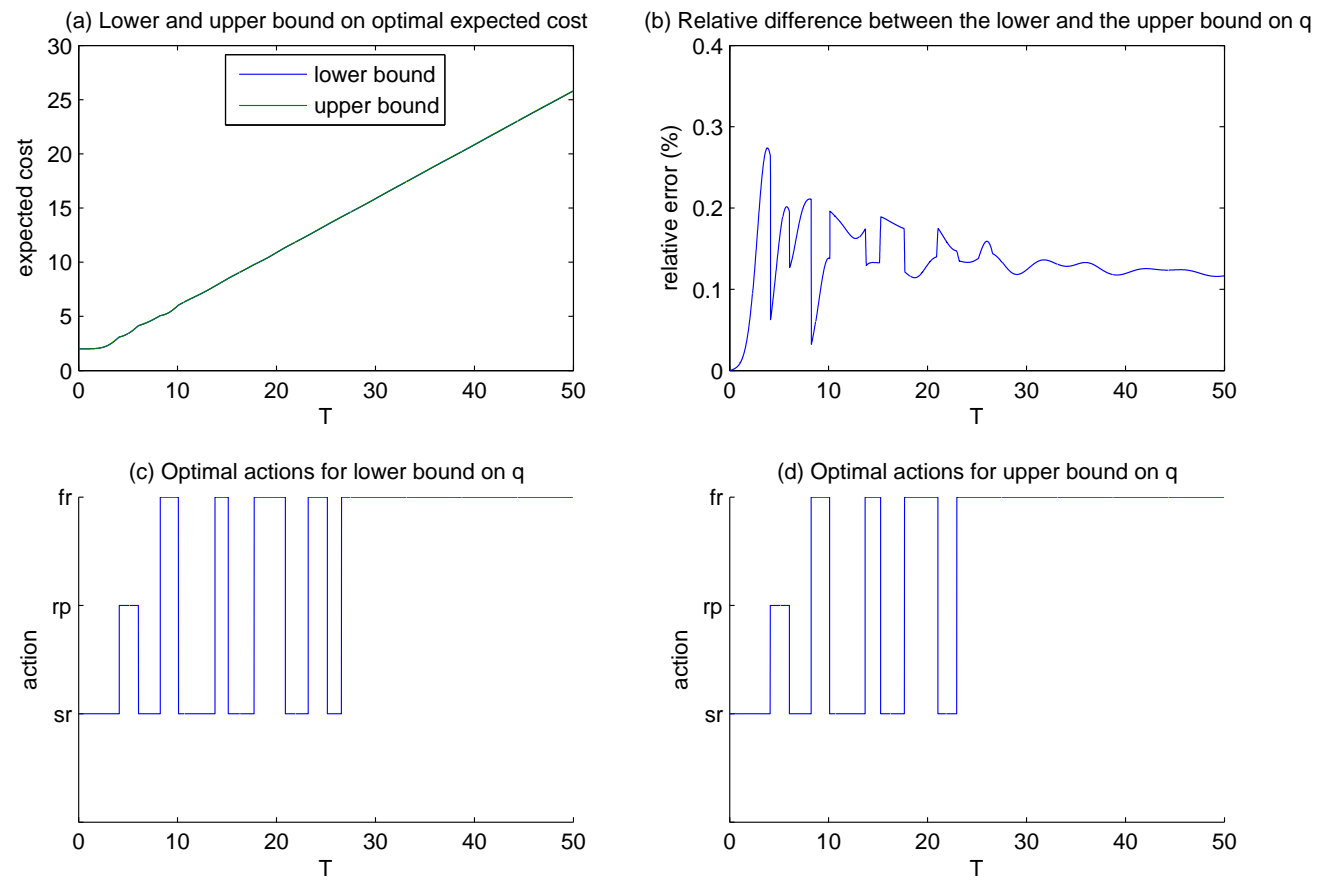

Figure 4: (a) Fixed points $\underline{q}$ and $\bar{q}$ of the operators $L_{h}$ and $U_{h}$, respectively. (b) Relative difference $100\left(\bar{q}\left(t_{n}\right)-q\left(t_{n}\right)\right) / \bar{q}\left(t_{n}\right)$. (c)-(d) Optimal maintenance actions associated with $\underline{q}$ and $\bar{q}$, respectively.

noteworthy that 'repainting' is cost optimal when $T \in[5,7]$ and that the optimal action changes a number of times from 'spot repair' to 'replacement' for $T \in[10,25]$. Here the end of horizon effect may play a role. The optimal maintenance actions associated with the bounds $q$ and $\bar{q}$ are almost the same, again showing that the discretization yields a good approximation.

\section{Conclusions}

The life-cycle management of steel structures involves decisions regarding the timing and the type of maintenance of protective coatings. In this paper we have presented a model for optimal maintenance of such coatings on steel structures. The deterioration of coatings is represented by the size of the area affected by corrosion and this is modelled by a non-stationary gamma 
process. Imperfect maintenance actions such as spot repair and repainting reduce this size by a random amount, whereas replacement reduces the size to zero. After maintenance the size again follows a non-stationary gamma process with possibly different parameters. It is assumed that maintenance is done as soon as the gamma process exceeds a fixed threshold and consequently the time between two maintenance actions is the first time a gamma process exceeds some nonnegative random threshold. The problem is to find the sequence of maintenance actions that minimizes the expected cost over a finite horizon. The continuous-time problem is formulated as a renewal-type optimality equation and it is solved by discretizing time. It is shown that the discretization yields an accurate approximation of the original problem. The outcomes of a numerical experiment suggest that different maintenance actions can be optimal over the decision horizon.

\section{References}

Abdel-Hameed, M. (1975), A gamma wear process, IEEE Transactions on Reliability, 24(2), 152153.

Bakker, J. D., H. J. van der Graaf, and J. M. van Noortwijk (1999), Model of lifetime-extending maintenance, in M. C. Forde (ed.), Proceedings of the 8th International Conference on Structural Faults and Repair, Engineering Technics Press, Edinburgh.

Çinlar, E., Z. P. Bazant, and E. Osman (1977), Stochastic process for extrapolating concrete creep, Journal of the Engineering Mechanics Division, 103(EM6), 1069-1088.

Doksum, K. A. and A. Hóyland (1992), Models for variable-stress accelerated life testing experiments based on Wiener processes and the inverse Gaussian distribution, Technometrics, 34, 74-82.

Frangopol, D. M., M. J. Kallen, and J. M. van Noortwijk (2004), Probabilistic models for life-cycle performance of deteriorating structures: review and future directions, Progress in Structural Engineering and Materials, 6(4), 197-212.

Frenk, J. B. G., R. Dekker, and M. Kleijn (1997), A unified treatment of single component replacement models, Mathematical Methods of Operations Research, 45, 437-454.

Frenk, J. B. G. and R. P. Nicolai (2007), Approximating the randomized hitting time distribution of a non-stationary gamma process, Tech. Rep. EI 2007-18, Econometric Institute.

Heutink, A., A. Van Beek, J. M. van Noortwijk, H. E. Klatter, and A. Barendregt (2004), Environment-friendly maintenance of protective paint systems at lowest costs, in XXVII FATIPEC Congres; 19-21 April 2004, Aix-en-Provence, Paris: AFTPVA.

Karlin, S. and H. M. Taylor (1975), A First Course in Stochastic Processes, San Diego: Academic Press. 
Kreyszig, E. (1978), Introductory Functional Analysis with Applications, Wiley, New York.

Newby, M. J. and R. Dagg (2004), Optimal inspection and perfect repair, IMA Journal of Management Mathematics, 15, 175-192.

Nicolai, R. P., R. Dekker, and J. M. van Noortwijk (2007), A comparison of models for measurable deterioration: an application to coatings on steel structures, Reliability Engineering and System Safety (forthcoming).

Protter, P. (1992), Stochastic Integration and Differential Equations (A new approach), Springer Verlag, New York.

Rudin, W. (1976), Principles of Mathematical Analysis, third edn., McGraw Hill, New York.

Steutel, F. W. and K. van Harn (2004), Infinite Divisibility and Probability Distributions on the Real Line, Marcel Dekker, New York.

van Noortwijk, J. M. (2007), A survey of the application of gamma processes in maintenance, Reliability Engineering \& System Safety, in press.

van Noortwijk, J. M. and H. E. Klatter (1999), Optimal inspection decisions for the block mats of the Eastern-Scheldt barrier, Reliability Engineering \& System Safety, 65(3), 203-211.

Whitmore, G. A. and F. Schenkelberg (1997), Modelling accelerated degradation data using Wiener diffusion with a time scale transformation, Lifetime Data Analysis, 3, 27-45.

Whittaker, E. T. and G. N. Watson (1958), Modern Analysis, fourth edn., Cambridge University Press, London.

\section{Appendix}

\section{A On the overshoot of a non-stationary gamma process}

In this appendix we give an upper bound on the expected overshoot $\mathbb{E}\left(\mathbf{W}_{v, \lambda}(r)\right)$ of a non-stationary gamma process for any $r>0$. By relation (4) we know that

$$
\mathbf{W}_{v, \lambda}(r) \stackrel{d}{=} \lambda^{-1} \mathbf{W}(\lambda r)
$$

with $\mathbf{W}(\lambda r)$ the overshoot at level $\lambda r$ of a standard gamma process and so we only need to determine an upper bound on the expected overshoot of a standard gamma process. Introduce for any $h>0$ the hitting time

$$
\mathbf{T}_{h}(r):=\min \{n h>0: \mathbf{X}(n h)>r\}
$$


with $\mathbf{X}$ a standard gamma process. By the definition of the hitting time $\mathbf{T}(r)$ of a standard gamma process it is obvious that

$$
\mathbf{T}(r) \leq \mathbf{T}_{h}(r)
$$

almost surely. Also, since the standard gamma process has independent and identically distributed increments the random variables $\mathbf{S}_{n}:=\mathbf{X}(n h)$ form a partial sum process and for this process we introduce the renewal process $\mathbf{N}_{h}:=\left\{\mathbf{N}_{h}(t): t \geq 0\right\}$ given by

$$
\mathbf{N}_{h}(t)=\sup \left\{n \in \mathbb{N} \cup\{0\}: \mathbf{S}_{n} \leq t\right\} .
$$

This shows by relations (45) and 46) that

$$
\mathbf{T}_{h}(r)=h\left(\mathbf{N}_{h}(r)+1\right)
$$

and since $\mathbf{X}$ has increasing sample paths we obtain by relation (46) that

$$
\begin{aligned}
\mathbf{W}(r) & =\mathbf{X}(\mathbf{T}(r))-r \\
& \leq \mathbf{X}\left(h\left(\mathbf{N}_{h}(r)+1\right)\right)-r \\
& =\mathbf{S}_{\mathbf{N}_{h}(r)+1}-r
\end{aligned}
$$

This implies using the well-known relation $\mathbb{E}\left(\mathbf{S}_{\mathbf{N}_{h}(r)+1}\right)=\mathbb{E}\left(\mathbf{N}_{h}(r)+1\right) \mathbb{E}(\mathbf{X}(h))$ that

$$
\mathbb{E}(\mathbf{W}(r)) \leq \mathbb{E}\left(\mathbf{N}_{h}(r)+1\right) \mathbb{E}(\mathbf{X}(h))-r .
$$

Since $\mathbf{X}(h) \sim \operatorname{gamma}(h, 1)$ we obtain

$$
\mathbb{E}(\mathbf{X}(h))=h \text { and } \mathbb{E}\left(\mathbf{X}^{2}(h)\right)=h+h^{2} .
$$

Hence by Lorden's inequality for the renewal function $t \mapsto \mathbb{E}\left(\mathbf{N}_{h}(t)\right)$ (for an elementary proof of this inequality see Frenk et al., 1997) we obtain

$$
\mathbb{E}\left(\mathbf{N}_{h}(r)+1\right) \leq \frac{r}{h}+\frac{h+h^{2}}{h^{2}}=\frac{r+1}{h}+1
$$

This implies by relation (48) that

$$
\mathbb{E}(\mathbf{W}(r)) \leq h+1
$$

for every $r>0$. Since this inequality holds holds for any $h>0$ we finally obtain that $\mathbb{E}(\mathbf{W}(r)) \leq$ 1 and so $\mathbb{E}\left(\mathbf{W}_{v, \lambda}(r)\right) \leq \lambda^{-1}$. This also implies that $\mathbb{E}\left(\mathbf{W}_{v, \lambda}(\mathbf{R})\right) \leq \lambda^{-1}$ for any random variable $\mathbf{R}$ independent of the gamma process. We may assume that in practice the expected reduction $\mathbb{E}\left(\mathbf{R}_{a}\right) \gg \lambda^{-1}$ for any action $a \in A$ and so this justifies that we do not consider the overshoot in our model. 


\section{B Dynamic programming algorithm}

The algorithm below simultaneously computes lower and upper bounds for the minimal expected maintenance costs $t_{n}$ time units before the end of the horizon, just before a maintenance action has to be selected, for $n=0,1, \ldots, N$.

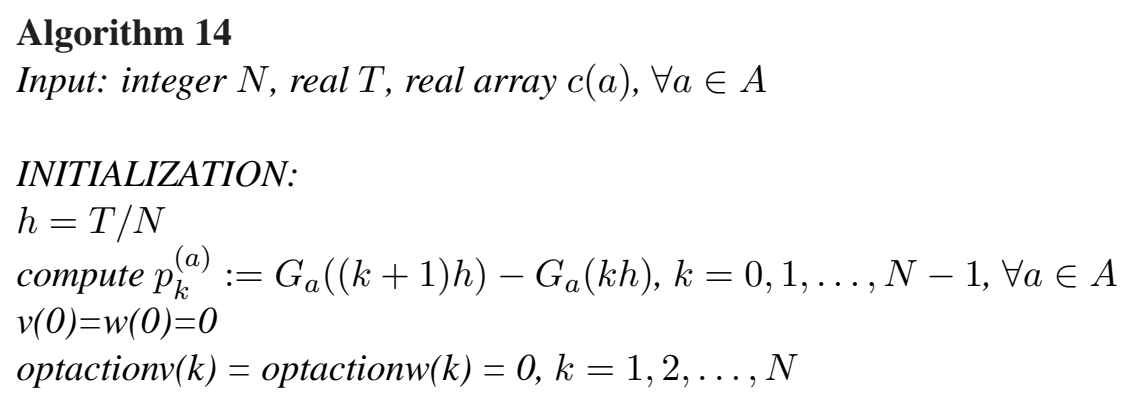

MAIN:

For $n=1$ to $N$ do

$\operatorname{sumv}(a)=\operatorname{sumw}(a)=0, \forall a \in A$

For $k=1$ to $n-1$ do

For $a \in A$ do

$\operatorname{sumv}(a)=\operatorname{sumv}(a)+v(n-k) p_{k-1}^{(a)}$

$\operatorname{sumw}(a)=\operatorname{sum} w(a)+w(n-k) p_{k}^{(a)}$

Next a

Next $k$

Let $v(n)=\min _{a \in A}\{c(a)+\operatorname{sumv}(a)\}$.

$\operatorname{optactionv}(n)=\arg \min _{a \in A}\{c(a)+\operatorname{sumv}(a)\}$.

Let $w(n)=\min _{a \in A}\left\{(c(a)+\operatorname{sumw}(a)) /\left(1-p_{0}^{(a)}\right)\right\}$.

$\operatorname{optactionw}(n)=\arg \min _{a \in A}\left\{(c(a)+\operatorname{sumw}(a)) /\left(1-p_{0}^{(a)}\right)\right\}$.

Next $n$

OUTPUT:

The arrays $v(n), n=0,1, \ldots, N$, and $w(n), n=0,1, \ldots, N$, consist of the lower and upper bounds on the minimal expected maintenance costs, $t_{n}$ time units before the end of the horizon and just before a maintenance action has to be selected. The elements of the arrays optactionv $(n)$, $n=1,2, \ldots, N$, and optactionw $(n), n=1,2, \ldots, N$, are the maintenance actions to be taken $t_{n}$ time units before the end of the horizon, just before the intervention level is exceeded. 